Objectives: To review the literature regarding different treatment modalities for $\mathrm{CHB}$

Methods: We performed a systematic review (August 2015) on Pubmed, using the following MeSH terms: "neonatal lupus", "congenital heart block"; results were restricted to human studies and English language. 1125 articles were assessed in abstract form and, after employing exclusion criteria, 267 original articles/case reports were evaluated in full text. Finally, 199 studies were included, reporting on a total of 492 CHB patients. All administered treatments were assessed on a patient-by-patient basis.

Results: A total of 243 cases reported data for CHB treatment: glucocorticoids (GCs) in 106 (43.6\%) cases, intravenous immunoglobulin (IVIG) in 14 (5.7\%) cases, and hydroxychloroquine in $5(2.0 \%)$ cases. 21 patients received plasmapheresis treatment. $134(55.1 \%)$ cases received no treatment. Both GCs and IVIG were mostly used in cases with complete atrioventricular (AV) block $(74.1 \%$ and $61.5 \%$ of cases, respectively). Different types of GCs were used: Dexamethasone in $54(58.6 \%)$ patients, prednisolone in $27(29.3 \%)$ and betamethasone in 11 $(11.9 \%)$ patients (total 92 patients with available data). Dosing schemes and regimens were also widely heterogeneous, with fifteen different regimens used by different centres (Table). Regarding IVIG treatment, six different algorithms were used. Similarly, five different plasmapheresis protocols were used.

Conclusions: There is no consensus in the treatment of $\mathrm{CHB}$. Drug selection and dosing regimens have wide heterogeneity. More than half cases received no treatment. Of note, prednisolone has often been used, despite its inability to cross the placenta.

Disclosure of Interest: None declared

DOI: 10.1136/annrheumdis-2017-eular.5909

\section{AB0472 IMPACT OF DISEASE ON FAMILY AND SOCIAL LIFE IN WOMEN WITH SYSTEMIC LUPUS ERYTHEMATOSUS (SLE)}

H. Babaoglu ${ }^{1}$, Ö. Varan ${ }^{1}$, S.C. Guven ${ }^{2}$, D. Tecer ${ }^{2}$, H. Küçük ${ }^{1}$, A. Tufan ${ }^{1}$, S. Haznedaroglu ${ }^{1}$, M.A. Öztürk ${ }^{1}$, B. Goker ${ }^{1}$. ${ }^{1}$ Department of Internal Medicine, Division of Rheumatology; ${ }^{2}$ Department of Physical and Rehabilitation Medicine, Division of Rheumatology, Gazi University Faculty of Medicine, Ankara, Turkey

Background: Systemic lupus erythematosus (SLE) is a chronic connective tissue disease with involvement of various organ systems and characteristically has a higher incidence in women than men ${ }^{1}$. The disease, as well as its treatment, could have significant effects on the quality of life of lupus patients.

Objectives: Here, we aimed to investigate the impact of SLE on quality of the social and family life of women.

Methods: One hundred and twenty women diagnosed with SLE were included in the study. A questionnaire including questions about family and social relations were applied and demographic information, educational status, marital status, organ involvement and treatment data were obtained. The results of this study are preliminary and the study is still ongoing.

Results: One hundred and twenty patients were studied. The average age was $37( \pm 10)$. 77 patients were married, 29 patients were single, 12 patients were divorced and 2 patients were widows. $29 \%$ of the patients were employed. $10,8 \%$ of the patients declared having difficulty in accepting their illnesses. $94,8 \%$ of the married patients had nuclear families. Relationship with partners and family members detoriated in $15,5 \%$ of the married patients after the diagnosis of SLE.

$10,7 \%$ of single patients ended their serious relationships and $28,6 \%$ developed negative thoughts about marriage after the diagnosis of SLE.

$17,6 \%$ of the married patients were exposed to verbal or physical violence by their partners. $33 \%$ of the patients declared having worse social life compared to prior to diagnosis and $20,7 \%$ declared having poor relations with their friends due to their disease. $34.5 \%$ of the patients stated that they received psychological counseling after their diagnosis. No significant relationship was found between family problems, social activities, age and educational level.

Conclusions: Systemic lupus erythematosus is a connective tissue disease affecting various organ systems and leading to various comorbidities. Our results suggest that family and social relations detoriate in lupus patients due to their illness independent of their age and educational level. Over one third of the patients received psychological counseling after diagnosis. Moreover, results of this study suggest that having a diagnosis of lupus has substantial impact on the marital considerations.

References:

[1] Hochberg, Marc C. Updating the American College of Rheumatology revised criteria for the classification of systemic lupus erythematosus. Arthritis \& Rheumatology 40.9 (1997): 1725-1725.

Disclosure of Interest: None declared

DOI: 10.1136/annrheumdis-2017-eular.6127

\section{AB0473 ANTI CCP AND ANTI MCV ANTIBODIES ARE MARKER OF ARTHRITIS IN SYSTEMIC LUPUS ERYTHEMATOUS \& SCLERODERMA}

A.M. Elsayed $^{1}$, S.A. ElBakry ${ }^{1}$, N.O. El-Azizi ${ }^{1}$, S.I. Ibrahim ${ }^{1}$, A. Abdelzaher ${ }^{1}$, N.M. Lotfy ${ }^{2}$, F.M. Badr ${ }^{1} .{ }^{1}$ Internal Medicine \& Rheumatology; ${ }^{2}$ Clinical pathology, Facilty of Medicine- Ain Shams University, Cairo, Egypt

Background: Anti-citrullinated protein antibodies (ACPA) have been reported as more specific serological markers of rheumatoid arthritis (RA). They provide a superior alternative to the rheumatoid factor (RF) test in laboratory diagnostics of RA (1). Different studies suggest that the enzymatic citrullination and the production of ACPAs may also be associated with other inflammatory arthritisassociated autoimmune diseases (2).

Objectives: Is to detect the presence of anti CCP and anti CMV antibodies in SLE \& SSc and its correlation to radiological findings and disease activity.

Methods: Our study was included 70 SLE patients and 30 systemic sclerosis (SSc) patients diagnosed according to ACR classification criteria. After informed consent, all patients were subjected to detailed history taking, full clinical examination including rheumatological examination, laboratory investigations: included CBC, ESR, CRP with titer, urine analysis, renal \& liver function, serum uric acid, Anti CCP antibodies \& Anti MCV antibodies by ELISA. X- ray and U/S on both hands and knees and disease activity score using SLEDAI score for SLE patients and SSc disease activity score (Medsgar score) for SSc patients.

Results: In our study, anti CCP antibodies were found in $8(11.4 \%)$ of SLE patients and $4(13.3 \%)$ SSc patients, while anti MCV antibodies were found in 14 (20\%) SLE patients and $8(26.7 \%)$ of SSc patients. There is association between presence of anti CCP Abs and anti MCV Abs and a clinically evident arthritis in both SLE and SSc. Strong relationship between high CRP level and a severe arthritis and joint erosions was noticed in SLE patients. A significant radiological evident in the form of synovial hypertrophy and bony erosions were found using ultrasonography and plain X-ray with seropositive anti CCP and anti MCV Abs in both SLE and SSc patients. In our study, cut off value of anti CCP which was $>12$, with sensitivity of $70.42 \%$, specificity of $60 \%$ positive predictive value of $80.6 \%$, negative predictive value of $46.2 \%$ with diagnostic accuracy of $61.7 \%$, and best cut off value of Anti MCV which was $>11$, with sensitivity of $98.46 \%$, specificity of $30 \%$, positive predictive value of $75.3 \%$, negative predictive value of $90 \%$ with diagnostic accuracy of $60.2 \%$ in SLE \& SSc.

Conclusions: There is a significant association between presence of anti CCP antibodies and anti MCV antibodies and the presence of evident arthritis either clinical or radiologically by using both $\mathrm{x}$-ray and $\mathrm{U} / \mathrm{S}$ on both hands and knees in SLE and SSc patients.

\section{References:}

[1] Xia L, Rulin J, Jinxia Z, et al. The Role of Anti-Mutated Citrullinated Vimentin Antibodies in the Diagnosis of Early Rheumatoid Arthritis. The Journal of Rheumatology 2009; 36:46.

[2] Szodoray P, Szabó Z, Kapitány A., et al. Anti-citrullinated protein/peptide autoantibodies in association with genetic and environmental factors as indicators of disease outcome in rheumatoid arthritis. 22 Autoimmunity Reviews 2010; 9: 140-3.

Acknowledgements: We thanks all the participating patients.

Disclosure of Interest: None declared

DOI: 10.1136/annrheumdis-2017-eular.2489

\section{AB0474 THYROID DYSFUNCTION IN SYSTEMIC LUPUS ERYTHEMATOSUS: ITS IMPACT AS A CARDIOVASCULAR RISK FACTOR}

A. Mabrook, M.I. Abd elkareem, M.M. Rayan. Rheumatology, Alazhr University, Assuit, Egypt

Background: Systemic lupus erythematosus (SLE) is an autoimmune disease caused by immune system-mediated tissue damage Thyroid function abnormalities and thyroid autoantibodies have been frequently described in patients with rheumatologic autoimmune diseases, such as SLE

Objectives: The aim of the study Was to assess thyroid function and anti-thyroid antibodies in SLE patients and evaulate the effects of the thyroid dysfunction on the clinical parameters, disease activity and assess its impact as a cardiovascular risk factor.

Methods: A total number of Fifty SLE female patients were selected, Triglycerides (TG), total cholesterol (TC), LDL and high density lipoprotein (HDL). Thyroid Stimulating Hormone (TSH), serum freeT3 (FT3), freeT4 (FT4).Serum thyroid peroxidase antibodies (TPOab) and serum thyroglobulin antibodies (TGab) erythrocyte sedimentation rate (ESR), C-reactive protein (CRP), IMT (intima-media thickness) of the carotid arteries and Echocardiography was done for all patients Results: There is a statistically significant correlation between SLE and thyroid disorder $\mathrm{p}$. value $<0.05$. clinical hypothyroidism was the most common abnormality presented followed by subclinical hypothyrodism. There is a statistically significant correlation between IMT and thyroid disorder $p$. value $<0.05$. There is a statistically significant correlation between cardiac valves disorder and thyroid disorderT, we found a statistically significant correlation between pericardial effusion and thyroid disorder $p$. value $<0.05$

Conclusions: we conclude that thyroid disorder is more common in lupus patients especially those in exacerbation and +ve for antithyroid Ab and Those with thyroid dysfunction had increased cardiovascular risk. These patients should be investigated for Lipid profile echocardiography and neck US for detection of early atherosclerosis and other CVD Those with thyroid dysfunction had increased cardiovascular risk.

References:

[1] Nakisa R, Mesbah Sh, Eskandar K-S, and Nazarinia M.A,(2015).

[2] The Prevalence of Thyroid Dysfunction in Patients With Systemic Lupus Erythematosus. Iran Red Crescent Med J. 2015 December; 17(12): e17298. 